\title{
Nonlinear Dynamics, Fixed Points and Coupled Fixed Points in Generalized Gauge Spaces with Applications to a System of Integral Equations
}

\begin{abstract}
Adrian Petruşel and Gabriela Petruşel
Faculty of Mathematics and Computer Science and Faculty of Business, Babeş-Bolyai University Cluj-Napoca, Kogălniceanu Street No. 1, 400084 Cluj-Napoca, Romania
\end{abstract}

Correspondence should be addressed to Adrian Petrușel; petrusel@math.ubbcluj.ro

Received 2 June 2015; Accepted 31 July 2015

Academic Editor: Gabriele Bonanno

Copyright (C) 2015 A. Petrușel and G. Petrușel. This is an open access article distributed under the Creative Commons Attribution License, which permits unrestricted use, distribution, and reproduction in any medium, provided the original work is properly cited.

We will discuss discrete dynamics generated by single-valued and multivalued operators in spaces endowed with a generalized metric structure. More precisely, the behavior of the sequence $\left(f^{n}(x)\right)_{n \in \mathbb{N}}$ of successive approximations in complete generalized gauge spaces is discussed. In the same setting, the case of multivalued operators is also considered. The coupled fixed points for mappings $t_{1}: X_{1} \times X_{2} \rightarrow X_{1}$ and $t_{2}: X_{1} \times X_{2} \rightarrow X_{2}$ are discussed and an application to a system of nonlinear integral equations is given.

\section{Introduction}

There are several generalizations of the Banach contraction principle. One of the most interesting ones was realized by Perov [1] (see also [2]), by replacing the context of a metric space with that of a space endowed with a vector-valued metric. Some contributions to fixed point theory in complete metric spaces in the sense of Perov can be found in [3-8] (for the case of single-valued operators) and, respectively, in [913] (for the case of multivalued operators).

Let $X$ be a nonempty set. A mapping $d: X \times X \rightarrow \mathbb{R}^{m}$ is called a vector-valued metric on $X$ if the following properties are satisfied: (a) $d(x, y) \geq O$ for all $x, y \in X$; if $d(x, y)=O$, then $x=y$ (where $O:=\underbrace{(0,0, \ldots, 0)})$

(b) $d(x, y)=d(y, x)$ for all $x, y \in X$;

(c) $d(x, y) \leq d(x, z)+d(z, y)$ for all $x, y \in X$.

Notice that, if $x, y \in \mathbb{R}^{m}, x=\left(x_{1}, \ldots, x_{m}\right)$, and $y=$ $\left(y_{1}, \ldots, y_{m}\right)$, then, by definition

$$
x \leq y \quad \text { iff } x_{i} \leq y_{i}, \text { for } i \in\{1,2, \ldots, m\} .
$$

Moreover, we will write

$$
\begin{aligned}
& x<y \quad \text { iff } x_{i} \leq y_{i} \text {, for } i \in\{1,2, \ldots, m\}, \text { there is } j \in\{1,2, \ldots, m\} \text { with } x_{j}<y_{j}, \\
& x \triangleleft y \quad \text { iff } x_{i}<y_{i} \text {, for } i \in\{1,2, \ldots, m\} .
\end{aligned}
$$

Notice that, through this paper, we will make an identification between row and column vectors in $\mathbb{R}^{m}$.
A nonempty set $X$ endowed with a vector-valued metric $d$ is called a generalized metric space in the sense of Perov 
(in short a generalized metric space) and it will be denoted by $(X, d)$. The notions of convergent sequence, Cauchy sequence, completeness, open and closed subset, open and closed ball, and so forth are similar to those for usual metric spaces.

Notice that the generalized metric space in the sense of Perov is a particular case of the cone metric spaces (or $K$ metric space) (see $[14,15])$.

On the other hand, in 1959, Marinescu [16] extended the Banach Contraction Principle to locally convex spaces and Colojoară [17] and Gheorghiu [18] did the same for the case of gauge spaces, while Knill [19] considered the framework of an uniform space. In 1971, Cain Jr. and Nashed [20] extended the notion of contraction to Hausdorff locally convex linear spaces. They showed that, on sequentially complete subset, the Banach contraction principle is still valid. In 2000, Frigon [21] introduced the notion of generalized contraction in gauge spaces and proved that every generalized contraction on a complete gauge space (sequentially complete gauge space) has a unique fixed point. For a nice survey on the same subject see also Frigon [22].

Definition 1. Let $X$ be any set. A mapping $p: X \times X \rightarrow \mathbb{R}_{+}$ is called a pseudometric (or a gauge) in $X$ whenever

(1) $p(x, y) \geq 0$, for all $x, y \in X$;

(2) $x=y$; then $p(x, y)=0$;

(3) $p(x, y)=p(y, x)$, for all $x, y \in \mathrm{X}$;

(4) $p(x, z) \leq p(x, y)+p(y, z)$, for every triple of point.

Definition 2. A family $\mathscr{P}=\left\{p_{\alpha}\right\}_{\alpha \in \Lambda}$ of pseudometrics on $X$ (or a gauge structure on $X$ ), where $\Lambda$ is a directed set, is said to be separating if for each pair of points $x, y \in X$, with $x \neq y$, there is a $p_{\alpha} \in \mathscr{P}$ such that $p_{\alpha}(x, y) \neq 0$.

A pair $(X, \mathscr{P})$ of a nonempty set $X$ and a separating gauge structure $\mathscr{P}$ on $X$ is called a gauge space.

It is well known (see [23, pages 198-204]) that any family $\mathscr{P}$ of pseudometrics on a set $X$ induces on $X$ a uniform structure $\mathcal{U}$ and, conversely, any uniform structure $\mathcal{U}$ on $X$ is induced by a family of pseudometrics on $X$. In addition, we have that $\mathcal{U}$ is separating (or Hausdorff) if and only if $\mathscr{P}$ is separating. Thus, we may identify gauge spaces and Hausdorff uniform spaces.

If map $p$ takes the values in $\mathbb{R}_{+}^{m}$ (i.e., $p: X \times X \rightarrow \mathbb{R}_{+}^{m}$ and satisfies the axioms of Definition 1 ), then it is called a vectorvalued gauge (or a generalized gauge) on $X$. In this case, the pair $(X, \mathscr{P})$ (where $\mathscr{P}=\left\{p_{\alpha}\right\}_{\alpha \in \Lambda}$ is a family of separating vector-valued gauges on $X$ ) is called a generalized gauge space; see [24]. The properties of the generalized gauge spaces (i.e., the notions of convergent sequences, Cauchy sequences and completeness, open and closed sets, etc.) are similar to those for gauge spaces.

An important concept in the study of different kinds of systems of operatorial equations is that of coupled fixed point. The concept of coupled fixed point for nonlinear operators was considered first by Opoitsev (see [25-27]) and then, in 1987, by Guo and Lakshmikantham (see [28]) in connection with coupled quasisolutions of an initial value problem for ordinary differential equations. A new research direction for the theory of coupled fixed points in ordered metric space was initiated by Bhaskar and Lakshmikantham in [29] and by Lakshmikantham and Ćirić in [30] using contraction type conditions on the operator. For other results on coupled fixed point theory see [29-33] and so forth.

In this paper, we will present fixed point and coupled fixed point theorems for single-valued and multivalued operators in spaces endowed with some generalized metrics. More precisely, the case of complete generalized gauge spaces is discussed. The dynamics of the sequence of successive approximations in each case is considered. An application to a nonlinear system of mixed integral equations is given.

\section{Preliminaries}

Let us recall first some important preliminary concepts and results.

We denote by $M_{m}\left(\mathbb{R}_{+}\right)$the set of all $m \times m$ matrices with positive elements, by $I$ the identity $m \times m$ matrix, and by $O$ the zero $m \times m$ matrix.

Definition 3. A $m \times m$ square matrix $A$ of real numbers is said to be convergent to zero if $A^{n} \rightarrow O$ as $n \rightarrow \infty$; see, for example [34].

A classical result in matrix analysis is the following theorem (see $[34,35])$.

Theorem 4. Let $A \in M_{m}\left(\mathbb{R}_{+}\right)$. The following assertions are equivalent:

(i) A is convergent towards zero;

(ii) its spectral radius $\rho(A)$ is strictly less than 1 ; that is, $|\lambda|<1$, for every $\lambda \in \mathbb{C}$ with $\operatorname{det}(A-\lambda I)=0$;

(iii) the matrix $(I-A)$ is nonsingular and

$$
(I-A)^{-1}=I+A+\cdots+A^{n}+\cdots ;
$$

(iv) the matrix $(I-A)$ is nonsingular and $(I-A)^{-1}$ has nonnegative elements;

(v) $A^{n} q \rightarrow O$ and $q A^{n} \rightarrow O$ as $n \rightarrow \infty$, for each $q \epsilon$ $\mathbb{R}^{m}$

(vi) the matrices $q A$ and $A q$ converge to $O$, for each $q \in$ $(1, Q)$, where $Q:=1 / \rho(A)$.

If $X$ is a nonempty set and $f: X \rightarrow X$ is an operator, then

$$
\operatorname{Fix}(f):=\{x \in X ; x=f(x)\} .
$$

We recall now Perov's fixed point theorem (see [1]; see also [2]).

Theorem 5 (Perov). Let $(X, d)$ be a complete generalized metric space and the operator $f: X \rightarrow X$ with the property that there exists a matrix $A \in M_{m}\left(\mathbb{R}_{+}\right)$convergent towards zero such that

$$
d(f(x), f(y)) \leq A d(x, y), \quad \forall x, y \in X .
$$


Then,

(1) $\operatorname{Fix}(f)=\left\{x^{*}\right\}$;

(2) the sequence of successive approximations $\left(x_{n}\right)_{n \in \mathbb{N}}$, $x_{n}:=f^{n}\left(x_{0}\right)$ is convergent in $X$ to $x^{*}$, for all $x_{0} \in X$;

(3) one has the following estimation:

$$
d\left(x_{n}, x^{*}\right) \leq A^{n}(I-A)^{-1} d\left(x_{0}, x_{1}\right) .
$$

Notice that in Precup [8] as well as in [4, 6] are pointed out the advantages of working with vector-valued norm with respect to the usual scalar norms.

There is a vast literature concerning this approach; see also, for example, $[3,5,7,8,36]$.

Let $(X, \mathscr{P})$ be a (generalized) gauge space. Then, a sequence $\left(x_{n}\right)_{n \in \mathbb{N}}$ of elements in $X$ is said to be Cauchy if, for every $\varepsilon>0$ and $\alpha \in A$, there is an $N$ with $p_{\alpha}\left(x_{n}, x_{n+p}\right) \leq \varepsilon$ for all $n \geq N$ and $p \in \mathbb{N}$. The sequence $\left(x_{n}\right)_{n \in \mathbb{N}}$ is called convergent if there exists an $x^{*} \in X$ such that, for every $\varepsilon>0$ and $\alpha \in A$, there is an $N$ with $p_{\alpha}\left(x^{*}, x_{n}\right) \leq \varepsilon$ for all $n \geq N$. We write $x_{n} \rightarrow x^{*}$ as $n \rightarrow \infty$.

Definition 6. A (generalized) gauge space is called sequentially complete if any Cauchy sequence is convergent. A subset of $X$ is said to be sequentially closed if it contains the limit of any convergent sequence of its elements.

If $(X, \mathscr{P})$ is a (generalized) gauge space, then $f: X \rightarrow X$ is continuous with respect to $\mathscr{P}$ if, for any sequence $\left(x_{n}\right)_{n \in \mathbb{N}}$ which converges (with respect to $\mathscr{P}$ ) to $x \in X$, we have that the sequence $\left(f\left(x_{n}\right)\right)_{n \in \mathbb{N}}$ converges (with respect to $\mathscr{P}$ ) to $f(x)$.

For further details see Dugundji [23] and Granas and Dugundji [37].

We will focus our attention on the following system of operatorial equations:

$$
\begin{aligned}
& x=t_{1}(x, y), \\
& y=t_{2}(x, y),
\end{aligned}
$$

where $t_{1}, t_{2}: X \times X \rightarrow X$ are two given single-valued operators.

By definition, a solution $(x, y) \in X \times X$ of the above system is called a coupled fixed point for the operators $t_{1}$ and $t_{2}$. Notice that if $s: X \times X \rightarrow X$ is an operator and we define

$$
\begin{aligned}
& t_{1}(x, y):=s(x, y), \\
& t_{2}(x, y):=s(y, x),
\end{aligned}
$$

then we get the classical concept of coupled fixed point for operator $s$ introduced by Opoitsev and then intensively studied in several papers by many authors.

The case of the coupled fixed point problem in the multivalued setting is defined as follows: find $(x, y) \in X \times X$ solution of the following system of operatorial inclusions:

$$
\begin{aligned}
& x \in T_{1}(x, y), \\
& y \in T_{2}(x, y),
\end{aligned}
$$

where $T_{1}, T_{2}: X \times X \rightarrow P(X)$ are two given multivalued operators.

The concept of coupled fixed point for a multivalued operator is accordingly defined.

\section{Fixed Point Theorems in Gauge Spaces}

Let us consider first the single-valued case. We will point out first the framework of our study.

If $\mathscr{P}=\left\{p_{\alpha}\right\}_{\alpha \in \Lambda}$ and $\mathbb{Q}=\left\{q_{\beta}\right\}_{\beta \in \Gamma}$ are two separating (generalized) gauge structures on a set $X$ (where $\Lambda$ and $\Gamma$ are directed sets), then for $r=\left\{r_{\beta}\right\}_{\beta \in \Gamma} \in\left(\mathbb{R}_{+}^{m}\right)^{\Gamma}$ (with $O \triangleleft r_{\beta}$ for every $\beta \in \Gamma)$ and $x_{0} \in X$ we will denote by ${\overline{\widetilde{B}_{q}\left(x_{0} ; r\right)}}^{p}$ the closure of $\widetilde{B}_{q}\left(x_{0} ; r\right)$ in $(X, \mathscr{P})$, where

$$
\widetilde{B}_{q}\left(x_{0} ; r\right)=\left\{x \in X: q_{\beta}\left(x_{0}, x\right) \leq r_{\beta}, \forall \beta \in \Gamma\right\} .
$$

In this case, the set ${\overline{\widetilde{B}_{q}\left(x_{0} ; r\right)}}^{p}$ is sequentially closed in $(X, \mathscr{P})$. If there is just one separating (generalized) gauge structure $\mathscr{P}=\left\{p_{\alpha}\right\}_{\alpha \in \Lambda}$ on $X$, then it is well known that $\widetilde{B}_{p}\left(x_{0} ; r\right)$ is sequentially closed in $(X, \mathscr{P})$.

We can prove the following local fixed point theorems.

Theorem 7. Let $X$ be a nonempty set endowed with two separating generalized gauge structures $\mathscr{P}=\left\{p_{\alpha}\right\}_{\alpha \in \Lambda}, \mathbb{Q}=$ $\left\{q_{\beta}\right\}_{\beta \in \Gamma}$ (where $\Lambda$ and $\Gamma$ are directed sets), $r=\left\{r_{\beta}\right\}_{\beta \in \Gamma} \in\left(\mathbb{R}_{+}^{m}\right)^{\Gamma}$ (with $O \triangleleft r_{\beta}$ for every $\beta \in \Gamma$ ), $x_{0} \in X$ and $f:{\overline{\widetilde{B}_{q}\left(x_{0} ; r\right)}}^{p} \rightarrow X$ a continuous operator with respect to $\mathscr{P}$. One supposes that the following hold.

(i) $(X, \mathscr{P})$ is a sequentially complete generalized gauge space.

(ii) There exist a function $\psi: \Lambda \rightarrow \Gamma$ and $C:=\left\{C_{\alpha}\right\}_{\alpha \in \Lambda} \in$ $\left(\mathbb{R}_{+}^{m}\right)^{\Lambda}$ (with $O \triangleleft C_{\alpha}$ for every $\alpha \in \Lambda$ ) such that

$p_{\alpha}(x, y) \leq C_{\alpha} \cdot q_{\psi(\alpha)}(x, y)$,

$$
\text { for every } \alpha \in \Lambda, x, y \in{\overline{\widetilde{B}_{q}\left(x_{0} ; r\right)}}^{p} \text {. }
$$

(iii) There exists a function $\varphi: \Gamma \rightarrow \Gamma$ and $M \in$ $\mathbb{M}_{m \times m}\left(\mathbb{R}_{+}\right)^{\Gamma}$ with $M:=\left\{M_{\beta}\right\}_{\beta \in \Gamma}$ such that, for every $\beta \in \Gamma$, the following implication holds:

$$
\begin{aligned}
x, y & \in{\widetilde{\widetilde{B}_{q}\left(x_{0} ; r\right)}}^{p} \\
& \Longrightarrow q_{\beta}(f(x), f(y)) \leq M_{\beta} q_{\varphi(\beta)}(x, y) .
\end{aligned}
$$

(iv) $\sum_{k=1}^{\infty} M_{\beta} M_{\varphi(\beta)} \cdots M_{\varphi^{k-1}(\beta)} q_{\varphi^{k}(\beta)}(u, v)<\infty$, for each $\beta \in \Gamma$ and every $u, v \in{\overline{\widetilde{B}_{q}\left(x_{0} ; r\right)}}^{p}$.

(v) If $r^{0}=\left\{r_{\beta}^{0}\right\}_{\beta \in \Gamma} \in\left(\mathbb{R}_{+}^{m}\right)^{\Gamma}$ (with $O \triangleleft r_{\beta}^{0}$ for every $\beta \in \Gamma$ ) is given by the expression $r_{\beta}^{0}:=\left(I-M_{\beta}\right)^{-1} q_{\beta}\left(x_{0}\right.$, $\left.f\left(x_{0}\right)\right)$, then $r_{\beta}^{0} \leq r_{\beta}$, for each $\beta \in \Gamma$. 
Then, $f$ has a unique fixed point $x^{*} \in{\overline{\widetilde{B}_{q}\left(x_{0} ; r^{0}\right)}}^{p}$ and the sequence $\left(f^{n}(x)\right)_{n \in \mathbb{N}}$ of successive approximations of $f$ converges to $x^{*}$, for any $x \in{\overline{\widetilde{B}_{q}\left(x_{0} ; r^{0}\right)}}^{p}$.

Proof. Notice first that the set ${\overline{\widetilde{B}_{q}\left(x_{0} ; r^{0}\right)}}^{p}$ is invariant with

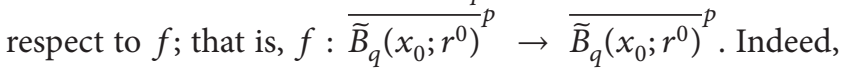
let $x \in{\overline{\widetilde{B}_{q}\left(x_{0} ; r^{0}\right)}}^{p}$. Then, there exists a sequence $\left(u_{n}\right)_{n \in \mathbb{N}}$ in $\widetilde{B}_{q}\left(x_{0} ; r^{0}\right)$ which converges (with respect to $\mathscr{P}$ ) to $x$. Since $f$ is continuous with respect to $\mathscr{P}$, we get that the sequence $\left(f\left(u_{n}\right)\right)_{n \in \mathbb{N}}$ converges (with respect to $\mathscr{P}$ ) to $f(x)$. So, we should show now that $f\left(u_{n}\right) \in \widetilde{B}_{q}\left(x_{0} ; r^{0}\right)$, for every $n \in \mathbb{N}$. Then, using the assumption (v), for each $\beta \in \Gamma$, we have

$$
\begin{aligned}
q_{\beta}\left(x_{0}, f\left(u_{n}\right)\right) \leq & q_{\beta}\left(f\left(u_{n}\right), f\left(x_{0}\right)\right) \\
& +q_{\beta}\left(x_{0}, f\left(x_{0}\right)\right) \\
\leq & M_{\beta} q_{\beta}\left(x_{0}, u_{n}\right)+q_{\beta}\left(x_{0}, f\left(x_{0}\right)\right) \\
\leq & M_{\beta} r_{\beta}^{0}+q_{\beta}\left(x_{0}, f\left(x_{0}\right)\right)=r_{\beta}^{0} .
\end{aligned}
$$

Now, in a classical manner (see, e.g., Theorem 2.1 in Novac and Precup [24]), we get that, for any $x \in{\overline{\widetilde{B}_{q}\left(x_{0} ; r^{0}\right)}}^{p}$, the sequence $\left(f^{n}(x)\right)_{n \in \mathbb{N}}$ is Cauchy in $(X, \mathcal{Q})$. By assumption (ii), the sequence is also Cauchy in $(X, \mathscr{P})$. Notice now that, since $(X, \mathscr{P})$ is a sequentially complete generalized gauge space, we have that $\left({\widetilde{\widetilde{B}_{q}\left(x_{0} ; r^{0}\right)}}^{p}, \mathscr{P}\right)$ is a sequentially complete generalized gauge space too. Thus, the sequence $\left(f^{n}(x)\right)_{n \in \mathbb{N}}$ is convergent (with respect to $\mathscr{P}$ ) to a certain element $x^{*} \in$ ${\overline{\widetilde{B}_{q}\left(x_{0} ; r^{0}\right)}}^{p}$. By the continuity of $f$ with respect to $\mathscr{P}$, we get that $x^{*}=f\left(x^{*}\right)$. The uniqueness follows from assumptions (iii) and (iv). Indeed, if $x^{*}$ and $u^{*}$ are two distinct fixed points of $f$, then, for each $\beta \in \Gamma$, we have

$$
\begin{aligned}
q_{\beta}\left(x^{*}, u^{*}\right) & =q_{\beta}\left(f\left(x^{*}\right), f\left(u^{*}\right)\right) \leq M_{\beta} q_{\beta}\left(x^{*}, u^{*}\right) \\
& \leq \cdots \\
& \leq M_{\beta} M_{\varphi(\beta)} \cdots M_{\varphi^{k-1}(\beta)} q_{\varphi^{k}(\beta)}\left(x^{*}, u^{*}\right) .
\end{aligned}
$$

Then, by (iv), we get that $q_{\beta}\left(x^{*}, u^{*}\right)=0$, for each $\beta \in \Gamma$. Since the family $\mathbb{Q}$ is separating, we obtain that $x^{*}=u^{*}$.

In particular, from the above proof, we can obtain the following result.

Theorem 8. Let $X$ be a nonempty set endowed with two separating generalized gauge structures $\mathscr{P}=\left\{p_{\alpha}\right\}_{\alpha \in \Lambda}, \mathbb{Q}=$ $\left\{q_{\beta}\right\}_{\beta \in \Gamma}$ (where $\Lambda$ and $\Gamma$ are directed sets), $r=\left\{r_{\beta}\right\}_{\beta \in \Gamma} \in\left(\mathbb{R}_{+}^{m}\right)^{\Gamma}$ (with $O \triangleleft r_{\beta}$ for every $\beta \in \Gamma$ ), $x_{0} \in X$ and $f:{\overline{\widetilde{B}_{q}\left(x_{0} ; r\right)}}^{p} \rightarrow X$ a continuous operator with respect to $\mathscr{P}$. One supposes that the following hold:

(i) $(X, \mathscr{P})$ is a sequentially complete generalized gauge space. (ii) There exist a function $\psi: \Lambda \rightarrow \Gamma$ and $C:=\left\{C_{\alpha}\right\}_{\alpha \in \Lambda} \in$ $\left(\mathbb{R}_{+}^{m}\right)^{\Lambda}$ (with $O \triangleleft C_{\alpha}$ for every $\alpha \in \Lambda$ ) such that

$p_{\alpha}(x, y) \leq C_{\alpha} \cdot q_{\psi(\alpha)}(x, y)$,

$$
\text { for every } \alpha \in \Lambda, x, y \in{\overline{\widetilde{B}_{q}\left(x_{0} ; r\right)}}^{p} \text {. }
$$

(iii) There exists a function $\varphi: \Gamma \rightarrow \Gamma$ and $M:=$ $\left\{M_{\beta}\right\}_{\beta \in \Gamma} \in \mathbb{M}_{m \times m}\left(\mathbb{R}_{+}\right)^{\Gamma}$ such that, for every $\beta \in \Gamma$, the following implication holds:

$$
\begin{aligned}
x, y & \in \overline{\widetilde{B}}_{q}\left(x_{0} ; r\right) \\
& \Longrightarrow q_{\beta}(f(x), f(y)) \leq M_{\beta} q_{\varphi(\beta)}(x, y) .
\end{aligned}
$$

(iv) $\sum_{k=1}^{\infty} M_{\beta} M_{\varphi(\beta)} \cdots M_{\varphi^{k-1}(\beta)}<\infty$, for each $\beta \in \Gamma$.

(v) If $r^{0}=\left\{r_{\beta}^{0}\right\}_{\beta \in \Gamma} \in\left(\mathbb{R}_{+}^{m}\right)^{\Gamma}$ (with $O \triangleleft r_{\beta}^{0}$ for every $\beta \in \Gamma$ ) is given by the expression $r_{\beta}^{0}:=\left(I-M_{\beta}\right)^{-1} q_{\beta}\left(x_{0}, f\left(x_{0}\right)\right)$, then $r_{\beta}^{0} \leq r_{\beta}$, for each $\beta \in \Gamma$.

(vi) There exists $S \in \mathbb{R}_{+}^{m}$ (with $O \triangleleft S$ ), such that $q_{\beta}\left(x_{0}, f\left(x_{0}\right)\right) \leq S$, for every $\beta \in \Gamma$.

Then, $f$ has a unique fixed point $x^{*} \in{\overline{\widetilde{B}_{q}\left(x_{0} ; r^{0}\right)}}^{p}$ and the sequence $\left(f^{n}\left(x_{0}\right)\right)_{n \in \mathbb{N}}$ of successive approximations of $f$ converges to $x^{*}$.

Notice that if, in particular, the operator $f$ in the previous results is a self-one on the whole space (i.e., $f: X \rightarrow X$ ), then we obtain some global fixed point theorems on a set $X$ with two separating gauge structures.

Let us also remark the following particular cases with respect to the function $\varphi: \Lambda \rightarrow \Lambda$ :

(1) if $\varphi=1_{\Gamma}$, then if the matrix $M_{\beta}$ converges to zero for each $\beta \in \Gamma$, then assumptions (iv) in Theorems 7 and 8 takes place;

(2) if $\varphi^{p}=\varphi$ for some $p \in \mathbb{N}$ with $p \geq 2$, then assumptions (iv) in Theorems 7 and 8 take place if we suppose that $M_{\varphi(\beta)} \cdots M_{\varphi^{p-1}(\beta)}$ converges to zero, for each $\beta \in \Gamma$.

Remark 9. The above results extend (to the case of nonselfoperators on a set endowed with two separating gauge structures) some fixed point theorems given in $[3,18,22,24]$ and so forth.

We illustrate the above remarks by the following consequence of the previous results.

Theorem 10. Let $X$ be a nonempty set endowed with two separating generalized gauge structures $\mathscr{P}=\left\{p_{\alpha}\right\}_{\alpha \in \Lambda}, \mathbb{Q}=$ $\left\{q_{\beta}\right\}_{\beta \in \Gamma}$ (where $\Lambda$ and $\Gamma$ are directed sets) and $f: X \rightarrow X$ a continuous operator with respect to $\mathscr{P}$. One supposes that the following hold:

(i) $(X, \mathscr{P})$ is a sequentially complete generalized gauge space. 
(ii) There exist a function $\psi: \Lambda \rightarrow \Gamma$ and $C:=\left\{C_{\alpha}\right\}_{\alpha \in \Lambda} \in$ $\left(\mathbb{R}_{+}^{m}\right)^{\Lambda}$ (with $O \triangleleft C_{\alpha}$ for every $\alpha \in \Lambda$ ) such that

$$
\begin{aligned}
& p_{\alpha}(x, y) \leq C_{\alpha} \cdot q_{\psi(\alpha)}(x, y), \\
& \qquad \text { for every } \alpha \in \Lambda, x, y \in{\overline{\widetilde{B}_{q}\left(x_{0} ; r\right)}}^{p} .
\end{aligned}
$$

(iii) There exists a function $\varphi: \Gamma \rightarrow \Gamma$ and $M:=$ $\left\{M_{\beta}\right\}_{\beta \in \Gamma} \in \mathbb{M}_{m \times m}\left(\mathbb{R}_{+}\right)^{\Gamma}$ such that, for every $\beta \in \Gamma$, the following implication holds:

$$
\begin{aligned}
x, y & \in X \\
& \Longrightarrow q_{\beta}(f(x), f(y)) \leq M_{\beta} q_{\varphi(\beta)}(x, y) .
\end{aligned}
$$

(iv) $\sum_{k=1}^{\infty} M_{\beta} M_{\varphi(\beta)} \cdots M_{\varphi^{k-1}(\beta)}<\infty$, for each $\beta \in \Gamma$.

(v) There exists $x_{0} \in X$ and $S \in \mathbb{R}_{+}^{m}$ (with $O \triangleleft S$ ), such that $q_{\beta}\left(x_{0}, f\left(x_{0}\right)\right) \leq S$, for every $\beta \in \Gamma$.

Then, $f$ has a unique fixed point $x^{*} \in X$ and the sequence $\left(f^{n}\left(x_{0}\right)\right)_{n \in \mathbb{N}}$ of successive approximations of $f$ converges to $x^{*}$.

A similar result, given for the cartesian product of two gauge spaces will be useful in applications.

Theorem 11. Let $X_{1}$ and $X_{2}$ be two nonempty sets endowed (resp.) with the separating generalized gauge structures $\mathscr{P}=$ $\left\{p_{\alpha}\right\}_{\alpha \in \Lambda}$ and, respectively, $\mathbb{Q}=\left\{q_{\beta}\right\}_{\beta \in \Gamma}$ (where $\Lambda$ and $\Gamma$ are directed sets) and denote $\tilde{d}_{\alpha, \beta}:\left(X_{1} \times X_{2}\right)^{2} \rightarrow \mathbb{R}_{+}^{2}$

$$
\widetilde{d}_{\alpha, \beta}((x, y),(u, v)):=\left(\begin{array}{l}
p_{\alpha}(x, u) \\
q_{\beta}(y, v)
\end{array}\right) .
$$

Let $f: X_{1} \times X_{2} \rightarrow X_{1} \times X_{2}$ be a continuous operator with respect to the product gauge structures $\mathscr{P} \times \mathbb{Q}$. We suppose that the following hold:

(i) $\left(X_{1}, \mathscr{P}\right)$ and $\left(X_{2}, \mathcal{Q}\right)$ are sequentially complete generalized gauge spaces.

(ii) There exists a function $\varphi: \Lambda \times \Gamma \rightarrow \Lambda \times \Gamma$ and $M:=$ $\left\{M_{\alpha, \beta}\right\}_{\alpha \in \Lambda, \beta \in \Gamma} \in \mathbb{M}_{2}\left(\mathbb{R}_{+}\right)^{\Lambda \times \Gamma}$ such that, for every $\alpha \in \Lambda$ and every $\beta \in \Gamma$, the following condition holds:

$$
\begin{aligned}
\tilde{d}_{\alpha, \beta}(f(z), f(w)) \leq M_{\alpha, \beta} \tilde{d}_{\varphi(\alpha), \varphi(\beta)} & (z, w), \\
& \forall z, w \in X_{1} \times X_{2} .
\end{aligned}
$$

(iii) $\sum_{k=1}^{\infty} M_{\alpha, \beta} M_{\varphi(\alpha), \varphi(\beta)} \cdots M_{\varphi^{k-1}(\alpha), \varphi^{k-1}(\beta)}<\infty$, for each $\alpha \in \Lambda$ and $\beta \in \Gamma$.

(iv) There exists $z_{0} \in X_{1} \times X_{2}$ and $s^{0} \in \mathbb{R}_{+}^{2}$ (with $0 \triangleleft s^{0}$ ), such that

$$
\widetilde{d}_{\alpha, \beta}\left(z_{0}, f\left(z_{0}\right)\right) \leq s^{0}
$$

Then, there exists a unique $z^{*}=\left(x^{*}, y^{*}\right) \in X_{1} \times X_{2}$ such that $z^{*}=f\left(z^{*}\right)$ and the sequence $f^{n}\left(z_{0}\right):=\left(f_{1}^{n}\left(z_{0}\right), f_{2}^{n}\left(z_{0}\right)\right)_{n \in \mathbb{N}}$ converges to $z^{*}$, where $f_{1}^{0}(x, y)=x, f_{2}^{0}(x, y)=y$ and

$$
\begin{aligned}
& f_{1}^{n}(z):=f_{1}^{n-1}\left(f_{1}(z), f_{2}(z)\right), \\
& f_{2}^{n}(z):=f_{2}^{n-1}\left(f_{1}(z), f_{2}(z)\right),
\end{aligned}
$$

for all $n \in \mathbb{N}^{*}$.
Moreover, for every $\alpha \in \Lambda$ and $\beta \in \Gamma$, we have the following estimation:

$$
\widetilde{d}_{\alpha, \beta}\left(f^{n}\left(z_{0}\right), z^{*}\right) \leq M^{n}(I-M)^{-1} \widetilde{d}_{\alpha, \beta}\left(z_{0}, f\left(z_{0}\right)\right) .
$$

Let us discuss now the multivalued case. Recall first that Frigon in [38] introduced a concept of multivalued admissible contraction and proved some interesting fixed point and continuation theorems on complete gauge spaces. We will present here some extensions of those results.

Let $(X, Q)$ (with $Q=\left\{q_{\beta}\right\}_{\beta \in \Gamma}$ ) be a gauge space and $P(X)$ the set of all nonempty subsets of $X$. For $Y \in P(X)$, we denote the diameter of the set $Y$ (for $\beta \in \Gamma$ ) by

$$
\operatorname{diam}_{\beta}(Y):=\sup \left\{q_{\beta}(x, y): x, y \in Y\right\} \text {. }
$$

We will also use the following symbols:

$$
\begin{aligned}
& P_{b}(X):=\{Y \in P(X): \underset{\beta}{\operatorname{diam}}(Y)<\infty, \forall \beta \in \Gamma\}, \\
& P_{c l}(X):=\{Y \in P(X): Y \text { is closed in }(X, Q)\} .
\end{aligned}
$$

The gap functional between two sets $Y$ and $Z$ from $(X, Q)$ is given by

$$
\begin{aligned}
& D_{\beta}: P(X) \times P(X) \longrightarrow \mathbb{R}_{+}, \\
& D_{\beta}(Y, Z)=\inf \left\{q_{\beta}(y, z) \mid y \in Y, z \in Z\right\}
\end{aligned}
$$

while the (generalized) Hausdorff-Pompeiu functional is defined by

$$
\begin{aligned}
& H_{\beta}: P(X) \times P(X) \longrightarrow \mathbb{R}_{+} \cup\{+\infty\}, \\
& H_{\beta}(Y, Z)=\max \left\{\sup _{y \in Y} D_{\beta}(y, Z), \sup _{z \in Z} D_{\beta}(Y, z)\right\} .
\end{aligned}
$$

The diameter (generalized) functional between two sets $Y$ and $Z$ from $(X, Q)$ is given by

$$
\begin{aligned}
& \delta_{\beta}: P(X) \times P(X) \longrightarrow \mathbb{R}_{+}+\cup\{+\infty\}, \\
& \delta_{\beta}(Y, Z)=\sup \left\{q_{\beta}(y, z) \mid y \in Y, z \in Z\right\} .
\end{aligned}
$$

If $F: X \rightarrow P(X)$ is a multivalued operator, then $x \in X$ is called fixed point for $F$ if and only if $x \in F(x)$. The set $\operatorname{Fix}(F):=\{x \in X \mid x \in F(x)\}$ is called the fixed point set of $F$. The symbol $\operatorname{Graph}(F):=\{(x, y) \in X \times X: y \in F(x)\}$ is the graph of the operator $F$.

Our first result in this direction is as follows.

Theorem 12. Let $X$ be a nonempty set endowed with two separating gauge structures $\mathscr{P}=\left\{p_{\alpha}\right\}_{\alpha \in \Lambda}, \mathbb{Q}=\left\{q_{\beta}\right\}_{\beta \in \Gamma}$ (where $\Lambda$ and $\Gamma$ are directed sets), $r=\left\{r_{\beta}\right\}_{\beta \in \Gamma} \in(0, \infty)^{\Gamma}, x_{0} \in X$ and

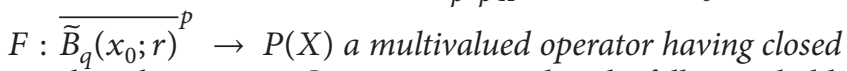
graph with respect to $\mathscr{P}$. One supposes that the following hold:

(i) $(X, \mathscr{P})$ is a sequentially complete gauge space. 
(ii) There exist a function $\psi: \Lambda \rightarrow \Gamma$ and $c:=\left\{c_{\alpha}\right\}_{\alpha \in \Lambda} \in$ $\mathbb{M}_{m \times m}(0, \infty)^{\Lambda}$ such that

$$
\begin{aligned}
& p_{\alpha}(x, y) \leq c_{\alpha} \cdot q_{\psi(\alpha)}(x, y) \\
& \qquad \text { for every } \alpha \in \Lambda, x, y \in{\overline{\widetilde{B}_{q}\left(x_{0} ; r\right)}}^{p} .
\end{aligned}
$$

(iii) There exists $k:=\left\{k_{\beta}\right\}_{\beta \in \Gamma} \in(0,1)^{\Gamma}$ such that, for every $\beta \in \Gamma$, the following implication holds:

$$
\begin{aligned}
x, y & \in{\overline{\widetilde{B}_{q}\left(x_{0} ; r\right)}}^{p} \\
& \Longrightarrow H_{\beta}(F(x), F(y)) \leq k_{\beta} q_{\beta}(x, y) .
\end{aligned}
$$

(iv) For every $x \in{\overline{\widetilde{B}_{q}\left(x_{0} ; r\right)}}^{p}$ and for every $\epsilon:=\left\{\epsilon_{\beta}\right\}_{\beta \in \Gamma} \epsilon$ $(0, \infty)^{\Gamma}$ there exists $y \in F(x)$ such that

$q_{\beta}(x, y) \leq D_{\beta}(x, F(x))+\epsilon_{\beta}, \quad$ for every $\beta \in \Gamma$.

(v) $D_{\beta}\left(x_{0}, F\left(x_{0}\right)\right)<\left(1-k_{\beta}\right) r_{\beta}$, for each $\beta \in \Gamma$.

Then, $F$ has at least one fixed point $x^{*} \in{\overline{\widetilde{B}_{q}\left(x_{0} ; r\right)}}^{p}$ and there exists a sequence $\left(x_{n}\right)_{n \in \mathbb{N}}$ of successive approximations of $F$ starting from $x_{0}$ which converges to an element $x^{*} \in \operatorname{Fix}(F)$.

Proof. By (v), we get that there exists $x_{1} \in F\left(x_{0}\right)$ such that $q_{\beta}\left(x_{0}, x_{1}\right)<\left(1-k_{\beta}\right) r_{\beta}$, for each $\beta \in \Gamma$. Obviously, $x_{1} \in$ $\widetilde{B}_{q}\left(x_{0} ; r\right)$. On the other hand, by (iv), there exists $x_{2} \in F\left(x_{1}\right)$ such that for every $\left\{\epsilon_{\beta}\right\}_{\beta \in \Gamma} \in(0, \infty)^{\Gamma}$ we have

$$
q_{\beta}\left(x_{1}, x_{2}\right) \leq D_{\beta}\left(x_{1}, F\left(x_{1}\right)\right)+\epsilon_{\beta}, \quad \text { for every } \beta \in \Gamma \text {. }
$$

Thus

$$
\begin{aligned}
q_{\beta}\left(x_{1}, x_{2}\right) & \leq H_{\beta}\left(F\left(x_{0}\right), F\left(x_{1}\right)\right)+\epsilon_{\beta} \\
& \leq k_{\beta} q_{\beta}\left(x_{0}, x_{1}\right)+\epsilon_{\beta} .
\end{aligned}
$$

Choosing $\epsilon_{\beta}:=k_{\beta}\left[\left(1-k_{\beta}\right) r_{\beta}-q_{\beta}\left(x_{0}, x_{1}\right)\right]$ we get that

$$
\begin{aligned}
q_{\beta}\left(x_{1}, x_{2}\right) \leq & k_{\beta} q_{\beta}\left(x_{0}, x_{1}\right) \\
& +k_{\beta}\left[\left(1-k_{\beta}\right) r_{\beta}-q_{\beta}\left(x_{0}, x_{1}\right)\right] \\
= & k_{\beta}\left(1-k_{\beta}\right) r_{\beta} .
\end{aligned}
$$

Moreover, $x_{2} \in \widetilde{B}_{q}\left(x_{0} ; r\right)$, since

$$
\begin{aligned}
q_{\beta}\left(x_{0}, x_{2}\right) & \leq q_{\beta}\left(x_{0}, x_{1}\right)+q_{\beta}\left(x_{1}, x_{2}\right) \\
& \leq\left(1-k_{\beta}\right) r_{\beta}+k_{\beta}\left(1-k_{\beta}\right) r_{\beta} \\
& =\left(1-k_{\beta}^{2}\right) r_{\beta} .
\end{aligned}
$$

By this procedure, we can obtain a sequence $\left(x_{n}\right)_{n \in \mathbb{N}}$ having the following properties:
(a) $x_{n+1} \in F\left(x_{n}\right)$, for each $n \in \mathbb{N}$;
(b) $x_{n} \in \widetilde{B}_{q}\left(x_{0} ; r\right)$, for every $n \in \mathbb{N}^{*}$;
(c) $q_{\beta}\left(x_{n}, x_{n+1}\right) \leq k_{\beta}^{n}\left(1-k_{\beta}\right) r_{\beta}$, for each $n \in \mathbb{N}^{*}$.

By a standard procedure, we obtain that $\left(x_{n}\right)_{n \in \mathbb{N}}$ is Cauchy in $(X, Q)$. Thus, by (ii) sequence $\left(x_{n}\right)_{n \in \mathbb{N}}$ is Cauchy in $(X, \mathscr{P})$ too. Notice now that (i) implies that $\left(\overline{\widetilde{B}}_{q}\left(x_{0} ; r\right), \mathscr{P}\right)$ is a sequentially complete gauge space. Thus, there exists $x^{*} \epsilon$ ${\overline{\widetilde{B}_{q}\left(x_{0} ; r\right)}}^{p}$ such that $\left(x_{n}\right) \rightarrow x^{*}$ as $n \rightarrow \infty$ (where the convergence is with respect to $\mathscr{P})$. By (b) and the closed graph hypothesis on $F$, we obtain that $x^{*} \in \operatorname{Fix}(F)$. The proof is now complete.

Remark 13. The above results extend (to the case of nonselfmultivalued operators on a set endowed with two separating gauge structures) some fixed point theorems given by Frigon; see $[21,22]$.

Notice that if, in particular, $F: X \rightarrow P(X)$, then we obtain a global fixed point theorem on a set $X$ with two separating gauge structures.

If, in the previous theorem, we replace (v) with a stronger condition, then we obtain the following result.

Theorem 14. Let $X$ be a nonempty set endowed with two separating gauge structures $\mathscr{P}=\left\{p_{\alpha}\right\}_{\alpha \in \Lambda}, \mathcal{Q}=\left\{q_{\beta}\right\}_{\beta \in \Gamma}$ (where $\Lambda$ and $\Gamma$ are directed sets), $r=\left\{r_{\beta}\right\}_{\beta \in \Gamma} \in(0, \infty)^{\Gamma}, x_{0} \in X$ and $F:{\overline{\widetilde{B}_{q}\left(x_{0} ; r\right)}}^{p} \rightarrow P(X)$ a multivalued operator having closed graph with respect to $\mathscr{P}$. One supposes that the following hold:

(i) $(X, \mathscr{P})$ is a sequentially complete gauge space.

(ii) There exist a function $\psi: \Lambda \rightarrow \Gamma$ and $c:=\left\{c_{\alpha}\right\}_{\alpha \in \Lambda} \in$ $\mathbb{M}_{m \times m}(0, \infty)^{\Lambda}$ such that

$p_{\alpha}(x, y) \leq c_{\alpha} \cdot q_{\psi(\alpha)}(x, y)$,

$$
\text { for every } \alpha \in \Lambda, x, y \in{\overline{\widetilde{B}_{q}\left(x_{0} ; r\right)}}^{p} \text {. }
$$

(iii) There exists $k:=\left\{k_{\beta}\right\}_{\beta \in \Gamma} \in(0,1)^{\Gamma}$ such that, for every $\beta \in \Gamma$, the following implication holds:

$$
\begin{aligned}
x, y & \in{\overline{\widetilde{B}_{q}\left(x_{0} ; r\right)}}^{p} \\
& \Longrightarrow H_{\beta}(F(x), F(y)) \leq k_{\beta} q_{\beta}(x, y) .
\end{aligned}
$$

(iv) For every $x \in{\overline{\widetilde{B}_{q}\left(x_{0} ; r\right)}}^{p}$ and for every $\epsilon:=\left\{\epsilon_{\beta}\right\}_{\beta \in \Gamma} \epsilon$ $(0, \infty)^{\Gamma}$ there exists $y \in F(x)$ such that

$q_{\beta}(x, y) \leq D_{\beta}(x, F(x))+\epsilon_{\beta}, \quad$ for every $\beta \in \Gamma$.

(v) $\delta_{\beta}\left(x_{0}, F\left(x_{0}\right)\right)<\left(1-k_{\beta}\right) r_{\beta}$, for each $\beta \in \Gamma$. 
Then, $F$ has at least one fixed point $x^{*} \in{\overline{\widetilde{B}_{q}\left(x_{0} ; r\right)}}^{p}$ and there exists a sequence $\left(x_{n}\right)_{n \in \mathbb{N}}$ of successive approximations of $F$ starting from any $x \in{\overline{\widetilde{B}_{q}\left(x_{0} ; r\right)}}^{p}$ which converges to an element $x^{*} \in \operatorname{Fix}(F)$.

Proof. Notice that (v) implies that $F:{\overline{\widetilde{B}_{q}\left(x_{0} ; r\right)}}^{p} \rightarrow$ $P\left({\overline{\widetilde{B}_{q}\left(x_{0} ; r\right)}}^{p}\right)$. Indeed, if $x \in{\overline{\widetilde{B}_{q}\left(x_{0} ; r\right)}}^{p}$, then there exists a sequence $u_{n} \in \widetilde{B}_{q}\left(x_{0} ; r\right)$ such that $u_{n} \rightarrow x$ as $n \rightarrow \infty$. Then, for any $y \in F(x)$ and for any $\beta \in \Gamma$, we have

$$
\begin{aligned}
q_{\beta}\left(x_{0}, y\right) \leq & \delta_{\beta}\left(x_{0}, F\left(x_{0}\right)\right)+H_{\beta}\left(F\left(x_{0}\right), F(x)\right) \\
\leq & \left(1-k_{\beta}\right) r_{\beta}+k_{\beta} q_{\beta}\left(x_{0}, x\right) \\
\leq & \left(1-k_{\beta}\right) r_{\beta} \\
& +k_{\beta}\left(q_{\beta}\left(x_{0}, u_{n}\right)+q_{\beta}\left(u_{n}, x\right)\right) \\
\leq & r_{\beta}+k_{\beta} q_{\beta}\left(u_{n}, x\right) \longrightarrow r_{\beta}, \quad \text { as } n \longrightarrow \infty .
\end{aligned}
$$

Thus $y \in \widetilde{B}_{q}\left(x_{0} ; r\right)$ and so $F(x) \subset{\widetilde{\widetilde{B}_{q}\left(x_{0} ; r\right)}}^{p}$. Now, the rest of the proof follows in a standard manner; see, for example, the proof of Theorem 7 and the proof of Theorem 6.2 in Frigon [22].

Remark 15. It is an open question to prove similar local fixed point results for multivalued operators on a set $X$ endowed with two separating generalized gauge structures.

\section{Existence, Uniqueness, and Stability for the Coupled Fixed Point Problem in Generalized Gauge Spaces}

We consider through this section the coupled fixed point problem in gauge spaces.

Let $X_{1}, X_{2}$ be nonempty sets endowed (resp.) with a separating gauge structure $\mathscr{P}=\left\{p_{\alpha}\right\}_{\alpha \in \Lambda}$ and, respectively, $\mathbb{Q}=\left\{q_{\beta}\right\}_{\beta \in \Gamma}$ (where $\Lambda$ and $\Gamma$ are directed sets).

We consider again the following system of operatorial equations:

$$
\begin{aligned}
& x=t_{1}(x, y), \\
& y=t_{2}(x, y),
\end{aligned}
$$

where $t_{1}: X_{1} \times X_{2} \rightarrow X_{1}$ and $t_{2}: X_{1} \times X_{2} \rightarrow X_{2}$ are two given single-valued operators. Using the above fixed point results, we can prove the following theorems.

Theorem 16. Let $\left(X_{1}, \mathscr{P}\right)$ and $\left(X_{2}, Q\right)$ be two complete generalized gauge spaces, and let $t_{1}: X_{1} \times X_{2} \rightarrow X_{1}$ and $t_{2}: X_{1} \times X_{2} \rightarrow X_{2}$ be two operators. Suppose there exists $\varphi: \Lambda \rightarrow \Lambda$ such that, for each $\alpha \in \Lambda$ and each $\beta \in \Gamma$, one has

$$
\begin{aligned}
& p_{\alpha}\left(t_{1}(x, y), t_{1}(u, v)\right) \\
& \quad \leq a_{\alpha, \beta} p_{\varphi(\alpha)}(x, u)+b_{\alpha, \beta} q_{\varphi(\beta)}(y, v), \\
& q_{\beta}\left(t_{2}(x, y), t_{2}(u, v)\right) \\
& \quad \leq c_{\alpha, \beta} p_{\varphi(\alpha)}(x, u)+d_{\alpha, \beta} q_{\varphi(\beta)}(y, v)
\end{aligned}
$$

for all $(x, y),(u, v) \in X_{1} \times X_{2}$ (where $a_{\alpha, \beta}, b_{\alpha, \beta}, c_{\alpha, \beta}, d_{\alpha, \beta} \in$ $\left.(0, \infty)^{\Lambda \times \Gamma}\right)$.

Suppose that $M:=M_{\alpha, \beta}=\left(\begin{array}{l}a_{\alpha, \beta} b_{\alpha, \beta} \\ c_{\alpha, \beta} d_{\alpha, \beta}\end{array}\right) \in M_{2}\left(\mathbb{R}_{+}\right)$has the property

(i) $\sum_{k=1}^{\infty} M_{\alpha, \beta} M_{\varphi(\alpha), \varphi(\beta)} \cdots M_{\varphi^{k-1}(\alpha), \varphi^{k-1}(\beta)}<\infty$, for each $\alpha \in \Lambda$ and $\beta \in \Gamma$,

and the following assumption takes also place:

(ii) there exists $\left(x_{0}, y_{0}\right) \in X_{1} \times X_{2}$ and $\left(s_{1}, s_{2}\right) \in \mathbb{R}_{+}^{2}$ (with $\left.s_{1}, s_{2}>0\right)$, such that

$$
\begin{aligned}
& p_{\alpha}\left(x_{0}, t_{1}\left(x_{0}, y_{0}\right)\right) \leq s_{1}, \\
& q_{\beta}\left(y_{0}, t_{2}\left(x_{0}, y_{0}\right)\right) \leq s_{2},
\end{aligned}
$$

for every $\alpha \in \Lambda$ and $\beta \in \Gamma$.

Then,

(i) there exists a unique element $z^{*}:=\left(x^{*}, y^{*}\right) \in X_{1} \times X_{2}$ such that

$$
\begin{aligned}
& x^{*}=t_{1}\left(x^{*}, y^{*}\right), \\
& y^{*}=t_{2}\left(x^{*}, y^{*}\right) ;
\end{aligned}
$$

(ii) the sequence $\left(t_{1}^{n}(x, y), t_{2}^{n}(x, y)\right)_{n \in \mathbb{N}}$ converges to $\left(x^{*}, y^{*}\right)$ as $n \rightarrow \infty$, where

$$
\begin{aligned}
& t_{1}^{n+1}(x, y):=t_{1}^{n}\left(t_{1}(x, y), t_{2}(x, y)\right), \\
& t_{2}^{n+1}(x, y):=t_{2}^{n}\left(t_{1}(x, y), t_{2}(x, y)\right),
\end{aligned}
$$

for all $n \in \mathbb{N}$;

(iii) we have the following estimation:

$$
\begin{aligned}
& \left(\begin{array}{l}
d_{1}\left(t_{1}^{n}\left(x_{0}, y_{0}\right), x^{*}\right) \\
d_{2}\left(t_{2}^{n}\left(x_{0}, y_{0}\right), y^{*}\right)
\end{array}\right) \\
& \quad \leq M^{n}(I-M)^{-1}\left(\begin{array}{l}
d_{1}\left(x_{0}, t_{1}\left(x_{0}, y_{0}\right)\right) \\
d_{2}\left(y_{0}, t_{2}\left(x_{0}, y_{0}\right)\right)
\end{array}\right) .
\end{aligned}
$$

Proof. (i)-(ii) Let us define again $t: X_{1} \times X_{2} \rightarrow X_{1} \times X_{2}$ by

$$
t(x, y)=\left(\begin{array}{l}
t_{1}(x, y) \\
t_{2}(x, y)
\end{array}\right) \text {. }
$$


Denote $Z:=X_{1} \times X_{2}$ and consider $\widetilde{d}_{\alpha, \beta}: Z \times Z \rightarrow \mathbb{R}_{+}^{2}$,

$$
\widetilde{d}_{\alpha, \beta}((x, y),(u, v)):=\left(\begin{array}{l}
p_{\alpha}(x, u) \\
q_{\beta}(y, v)
\end{array}\right) .
$$

Then we have

$$
\begin{gathered}
\widetilde{d}_{\alpha, \beta}(t(x, y), t(u, v))=\left(\begin{array}{l}
p_{\alpha}\left(t_{1}(x, y), t_{1}(u, v)\right) \\
q_{\beta}\left(t_{2}(x, y), t_{2}(u, v)\right)
\end{array}\right) \\
\leq\left(\begin{array}{l}
a_{\alpha, \beta} p_{\varphi(\alpha)}(x, u)+b_{\alpha, \beta} q_{\varphi(\beta)}(y, v) \\
c_{\alpha, \beta} p_{\varphi(\alpha)}(x, u)+d_{\alpha, \beta} q_{\varphi(\beta)}(y, v)
\end{array}\right) \\
=\left(\begin{array}{ll}
a_{\alpha, \beta} & b_{\alpha, \beta} \\
c_{\alpha, \beta} & d_{\alpha, \beta}
\end{array}\right)\left(\begin{array}{l}
p_{\varphi(\alpha)}(x, u) \\
q_{\varphi(\beta)}(y, v)
\end{array}\right) \\
=M_{\alpha, \beta} \cdot \widetilde{d}_{\varphi(\alpha), \varphi(\beta)}((x, y),(u, v)) .
\end{gathered}
$$

If we denote $(x, y):=z,(u, v):=w$, we get that

$$
d_{\alpha, \beta}(t(z), t(w)) \leq S \cdot d_{\varphi(\alpha), \varphi(\beta)}(z, w) .
$$

Applying Theorem 11, we get that there exists a unique element $\left(x^{*}, y^{*}\right) \in Z$ such that

$$
\left(x^{*}, y^{*}\right)=t\left(x^{*}, y^{*}\right)
$$

and is equivalent with

$$
\begin{aligned}
& x^{*}=t_{1}\left(x^{*}, y^{*}\right), \\
& y^{*}=t_{2}\left(x^{*}, y^{*}\right) .
\end{aligned}
$$

Moreover, for each $z \in Z$, we have that $t^{n}(z) \rightarrow z^{*}$ as $n \rightarrow$ $\infty$, where

$$
\begin{aligned}
t^{0}(z) & :=z, \\
t^{1}(z) & =t(x, y)=\left(t_{1}(x, y), t_{2}(x, y)\right), \\
t^{n+1}(z) & =\left(t_{1}^{n+1}(z), t_{2}^{n+1}(z)\right),
\end{aligned}
$$

where

$$
\begin{aligned}
& t_{1}^{n+1}(x, y):=t_{1}^{n}\left(t_{1}(x, y), t_{2}(x, y)\right), \\
& t_{2}^{n+1}(x, y):=t_{2}^{n}\left(t_{1}(x, y), t_{2}(x, y)\right) .
\end{aligned}
$$

Thus, for all $(x, y) \in Z$, we have that

$$
\begin{aligned}
& t_{1}^{n}(x, y) \longrightarrow x^{*} \quad \text { as } n \longrightarrow \infty, \\
& t_{2}^{n}(x, y) \longrightarrow y^{*} \text { as } n \longrightarrow \infty .
\end{aligned}
$$

As an application of the above results, we establish an existence and uniqueness theorem for a system of nonlinear integral equations on the real axis.
Theorem 17. Consider the following system:

$$
\begin{aligned}
& x(t)=\int_{0}^{t} f_{1}(s, x(s), y(s)) d s, \\
& y(t)=\int_{t-1}^{t} f_{2}(s, x(s), y(s)) d s,
\end{aligned}
$$

for $t \in \mathbb{R}_{+}$, where

$$
\begin{aligned}
& x(t)=\Phi(t), \\
& y(t)=\Psi(t),
\end{aligned}
$$

$$
t \in[-1,0] \text {. }
$$

Suppose that the following assumptions take place:

(i) the functions $f_{1}:[0, \infty) \times \mathbb{R}^{2} \rightarrow \mathbb{R}$ and $f_{2}:[-1, \infty) \times$ $\mathbb{R}^{2} \rightarrow \mathbb{R}$ are continuous;

(ii) there exists $k_{1}, l_{1}>0$ and $k_{2}, l_{2} \in L^{1}\left([0, \infty), \mathbb{R}_{+}\right)$such that

$$
\begin{aligned}
& (\text { ii })_{a}\left|f_{1}(s, x, y)-f_{1}(s, u, v)\right| \\
& \leq k_{1}|x-u|+l_{1}|y-v|, \\
& (i i)_{b}\left|f_{2}(s, x, y)-f_{2}(s, u, v)\right| \\
& \leq k_{2}(s)|x-u|+l_{2}(s)|y-v|,
\end{aligned}
$$

for everys $\in[0, \infty)$ and $x, y, u, v \in \mathbb{R}$;

(iii) for every $n \in \mathbb{N}^{*}$ the matrix

$$
M_{n}:=\left(\begin{array}{cc}
\frac{k_{1}}{\tau} & \frac{l_{1}}{\tau} \\
e^{\tau n} \int_{0}^{n} k_{2}(s) d s & \int_{0}^{n} l_{2}(s) d s
\end{array}\right)
$$

(where $\tau=\tau(n)>0$ can be arbitrary chosen) converges to zero;

(iv) the functions $\Phi, \Psi:[-1,0] \rightarrow \mathbb{R}$ are continuous.

Then, there exists a unique solution of system (55).

Proof. Let $X_{1}:=C[0, \infty)$ endowed with the family of gauges

$$
\|x\|_{n}^{C}:=\max _{t \in[0, n]}|x(t)|
$$

and $X_{2}:=C[0, \infty)$ endowed with the family of gauges

$$
\|x\|_{n}^{B}:=\max _{t \in[0, n]}|x(t)| e^{-\tau t}
$$

where $\tau>0$.

We consider the operators $t_{1}: X_{1} \times X_{2} \rightarrow X_{1}$ and $t_{2}:$ $X_{1} \times X_{2} \rightarrow X_{2}$ given by

$$
\begin{aligned}
& t_{1}(x, y)(t):=\int_{0}^{t} f_{1}(s, x(s), y(s)) d s, \\
& t_{2}(x, y)(t):=\int_{t-1}^{t} f_{2}(s, \tilde{x}(s), \tilde{y}(s)) d s,
\end{aligned}
$$


where

$$
\begin{aligned}
& \tilde{x}(t):= \begin{cases}\Phi(t), & t \in[-1,0] \\
x(t), & t \geq 0,\end{cases} \\
& \tilde{y}(t):= \begin{cases}\Psi(t), & t \in[-1,0] \\
y(t), & t \geq 0 .\end{cases}
\end{aligned}
$$

Then we have

$$
\begin{aligned}
& \left|t_{1}(x, y)(t)-t_{1}(u, v)(t)\right| \\
& \quad \leq \int_{0}^{t}\left|f_{1}(s, x(s), y(s))-f_{1}(s, u(s), v(s))\right| d s \\
& \quad \leq \int_{0}^{t}\left[k_{1}|x(s)-u(s)|+l_{1}|y(s)-v(s)|\right] d s \\
& \quad \leq \frac{k_{1}}{\tau}\|x-u\|_{n}^{B} e^{\tau t}+\frac{l_{1}}{\tau}\|y-v\|_{n}^{B} e^{\tau t} .
\end{aligned}
$$

Thus

$$
\left\|t_{1}(x, y)-t_{1}(u, v)\right\|_{n}^{B} \leq \frac{k_{1}}{\tau}\|x-u\|_{n}^{B}+\frac{l_{1}}{\tau}\|y-v\|_{n}^{B} .
$$

Since $\|\cdot\|_{n}^{B} \leq\|\cdot\|_{n}^{C}$, we obtain that

$$
\left\|t_{1}(x, y)-t_{1}(u, v)\right\|_{n}^{B} \leq \frac{k_{1}}{\tau}\|x-u\|_{n}^{B}+\frac{l_{1}}{\tau}\|y-v\|_{n}^{C} .
$$

For the second operator, for $t \in[0, n]$, we successively have

$$
\begin{aligned}
& \left|t_{2}(x, y)(t)-t_{2}(u, v)(t)\right| \\
& \quad \leq \int_{0}^{n}\left|f_{2}(s, x(s), y(s))-f_{2}(s, u(s), v(s))\right| d s \\
& \quad \leq \int_{0}^{n}\left[k_{2}(s)|x(s)-u(s)|+l_{2}(s)|y(s)-v(s)|\right] d s \\
& \leq\|x-u\|_{n}^{C} \cdot \int_{0}^{n} k_{2}(s) d s+\|y-v\|_{n}^{C} \cdot \int_{0}^{n} l_{2}(s) d s \\
& \leq\|x-u\|_{n}^{B} \cdot e^{\tau n} \int_{0}^{n} k_{2}(s) d s+\|y-v\|_{n}^{C} \\
& \quad \cdot \int_{0}^{n} l_{2}(s) d s .
\end{aligned}
$$

Thus, taking the maximum over $t \in[0, n]$, we get

$$
\begin{aligned}
& \left\|t_{2}(x, y)-t_{2}(u, v)\right\|_{n}^{C} \\
& \leq e^{\tau n} \int_{0}^{n} k_{2}(s) d s \cdot\|x-u\|_{n}^{B}+\int_{0}^{n} l_{2}(s) d s \\
& \cdot\|y-v\|_{n}^{C} .
\end{aligned}
$$

The conclusion of our theorem follows now by Theorem 16, for $\varphi(n)=n$.
Remark 18. For example, if $k_{2} \equiv 0$, then a sufficient condition for the convergence to zero of the matrix $M_{n}$, for every $n \in$ $\mathbb{N}^{*}$, is the convergence to zero of the matrix

$$
M:=\left(\begin{array}{cc}
\frac{k_{1}}{\tau} & \frac{l_{1}}{\tau} \\
0 & \left\|l_{2}\right\|_{L^{1}([0, \infty))}
\end{array}\right) .
$$

This last condition is, for example, satisfied (see [8]) if $\max \left\{k_{1} / \tau,\left\|l_{2}\right\|_{L^{1}([0, \infty))}\right\}<1$.

\section{Conflict of Interests}

The authors declare that there is no conflict of interests regarding the publication of this paper.

\section{Acknowledgment}

For the first author this work was supported by the research grant GSCE offered by Babeş-Bolyai University Cluj-Napoca, no. 30248/22.01.2015.

\section{References}

[1] A. I. Perov, "On the Cauchy problem for a system of ordinary differential equations," Pviblizhen. Met. Reshen. Differ. Uravn, vol. 2, pp. 115-134, 1964.

[2] A. I. Perov and A. V. Kibenko, "On a certain general method for investigation of boundary value problems," Izvestiya Akademii Nauk SSSR. Seriya Matematicheskaya, vol. 30, pp. 249-264, 1966 (Russian).

[3] R. P. Agarwal, "Contraction and approximate contraction with an application to multipoint boundary value problems," Journal of Computational and Applied Mathematics, vol. 9, no. 4, pp. 315-325, 1983.

[4] A.-D. Filip and A. Petruşel, "Fixed point theorems on spaces endowed with vector-valued metrics," Fixed Point Theory and Applications, vol. 2010, Article ID 281381, 15 pages, 2010.

[5] D. O'Regan, N. Shahzad, and R. P. Agarwal, "Fixed point theory for generalized contractive maps on spaces with vector-valued metrics," in Fixed Point Theory and Applications, pp. 143-149, Nova Science Publishers, New York, NY, USA, 2007.

[6] R. Precup and A. Viorel, "Existence results for systems of nonlinear evolution equations," International Journal of Pure and Applied Mathematics, vol. 47, no. 2, pp. 199-206, 2008.

[7] R. Precup and A. Viorel, "Existence results for systems of nonlinear evolution inclusions," Fixed Point Theory, vol. 11, no. 2, pp. 337-346, 2010.

[8] R. Precup, "The role of matrices that are convergent to zero in the study of semilinear operator systems," Mathematical and Computer Modelling, vol. 49, no. 3-4, pp. 703-708, 2009.

[9] A. Petrușel, "Multivalued weakly Picard operators and applications," Scientiae Mathematicae Japonicae, vol. 59, no. 1, pp. 169202, 2004.

[10] I.-R. Petre and A. Petruşel, "Krasnoselskii's theorem in generalized Banach spaces and applications," Electronic Journal of Qualitative Theory of Differential Equations, no. 85, pp. 1-20, 2012.

[11] Sh. Rezapour and P. Amiri, "Some fixed point results for multivalued operators in generalized metric spaces," Computers 
\& Mathematics with Applications, vol. 61, no. 9, pp. 2661-2666, 2011.

[12] A. Petruşel, C. Urs, and O. Mleşniţe, "Vector-valued metrics in fixed point theory," Contemporary Mathematics, vol. 636, pp. 149-164, 2015.

[13] A. Petruşel, G. Petruşel, and C. Urs, "Vector-valued metrics, fixed points and coupled fixed points for nonlinear operators," Fixed Point Theory and Applications, vol. 2013, article 218, 2013.

[14] P. P. Zabrejko, "K-metric and K-normed linear spaces: survey," Collectanea Mathematica, vol. 48, no. 4-6, pp. 825-859, 1997.

[15] L.-G. Huang and X. Zhang, "Cone metric spaces and fixed point theorems of contractive mappings," Journal of Mathematical Analysis and Applications, vol. 332, no. 2, pp. 1467-1475, 2007.

[16] G. Marinescu, Spaţii Vectoriale Topologice şi Pseudotopologice, vol. 4, Biblioteca Matematică, Editura Academiei Republicii Populare Romîne, Bucharest, Romania, 1959.

[17] I. Colojoară, "On a fixed point theorem in complete uniform spaces,” Com. Acad. R.P.R., vol. 11, pp. 281-283, 1961.

[18] N. Gheorghiu, "Contraction theoremin uniform spaces," Studii şi Cercetări Matematice, vol. 19, pp. 119-122, 1967 (Romanian).

[19] R. J. Knill, "Fixed points of uniform contractions," Journal of Mathematical Analysis and Applications, vol. 12, pp. 449-455, 1965.

[20] J. L. Cain Jr. and M. Z. Nashed, "Fixed points and stability for a sum of two operators in locally convex spaces," Pacific Journal of Mathematics, vol. 39, pp. 581-592, 1971.

[21] M. Frigon, "Fixed point results for generalized contractions in gauge spaces and applications," Proceedings of the American Mathematical Society, vol. 128, no. 10, pp. 2957-2965, 2000.

[22] M. Frigon, "Fixed point and continuation results for contractions in metric and gauge spaces," Fixed Point Theory and Its Applications, vol. 77, pp. 89-114, 2007.

[23] J. Dugundji, Topology, Allyn \& Bacon, Boston, Mass, USA, 1966.

[24] A. Novac and R. Precup, "Perov type results in gauge spaces and their applications to integral systems on semi-axis," Mathematica Slovaca, vol. 64, no. 4, pp. 961-972, 2014.

[25] V. I. Opoitsev, "Heterogeneous and combined concave operators," Siberian Mathematical Journal, vol. 16, no. 4, pp. 597-605, 1975 (Russian).

[26] V. I. Opoitsev, "Dynamics of collective behavior. III. Heterogenic systems," Avtomatika i Telemekhanika, vol. 36, pp. 124138, 1975 (Russian).

[27] V. I. Opoitsev and T. A. Khurodze, "Nonlinear operators in spaces with a cone," Tbilisskiü Gosudarstvennyü Universitet, Tbilisi, vol. 271, 1984 (Russian).

[28] D. J. Guo and V. Lakshmikantham, "Coupled fixed points of nonlinear operators with applications," Nonlinear Analysis. Theory, Methods \& Applications, vol. 11, no. 5, pp. 623-632, 1987.

[29] T. G. Bhaskar and V. Lakshmikantham, "Fixed point theorems in partially ordered metric spaces and applications," Nonlinear Analysis: Theory, Methods \& Applications, vol. 65, no. 7, pp. 13791393, 2006.

[30] V. Lakshmikantham and L. Ćirić, "Coupled fixed point theorems for nonlinear contractions in partially ordered metric spaces," Nonlinear Analysis: Theory, Methods \& Applications, vol. 70, no. 12, pp. 4341-4349, 2009.

[31] D. Guo, Y. J. Cho, and J. Zhu, Partial Ordering Methods in Nonlinear Problems, Nova Science Publishers, Hauppauge, NY, USA, 2004.
[32] S. Hong, "Fixed points for mixed monotone multivalued operators in Banach spaces with applications," Journal of Mathematical Analysis and Applications, vol. 337, no. 1, pp. 333-342, 2008.

[33] V. Berinde, "Generalized coupled fixed point theorems for mixed monotone mappings in partially ordered metric spaces," Nonlinear Analysis, vol. 74, no. 18, pp. 7347-7355, 2011.

[34] R. S. Varga, Matrix Iterative Analysis, vol. 27 of Springer Series in Computational Mathematics, Springer, Berlin, Germany, 2000.

[35] G. Allaire and S. M. Kaber, Numerical Linear Algebra, Springer, New York, NY, USA, 2008.

[36] A. Petruşel and I. A. Rus, "The theory of a metric fixed point theorem for multivalued operators," in Fixed Point Theory and Its Applications, L. J. Lin, A. Petruşel, and H. K. Xu, Eds., pp. 167-176, Yokohama, 2010.

[37] A. Granas and J. Dugundji, Fixed Point Theory, Springer, Berlin, Germany, 2003.

[38] M. Frigon, "Fixed point results for multivalued contractions on gauge spaces," in Set-Valued Mappings with Applications in Nonlinear Analysis, vol. 4 of Mathematical Analysis and Applications, pp. 175-181, Taylor \& Francis, London, UK, 2002. 


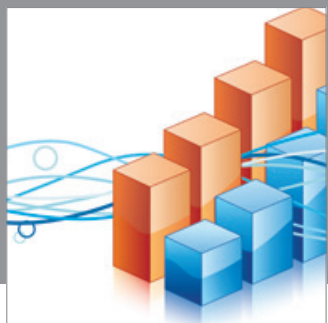

Advances in

Operations Research

mansans

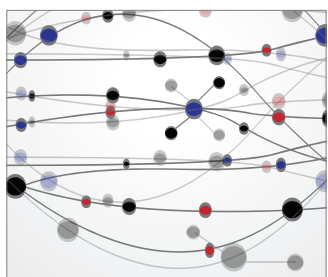

The Scientific World Journal
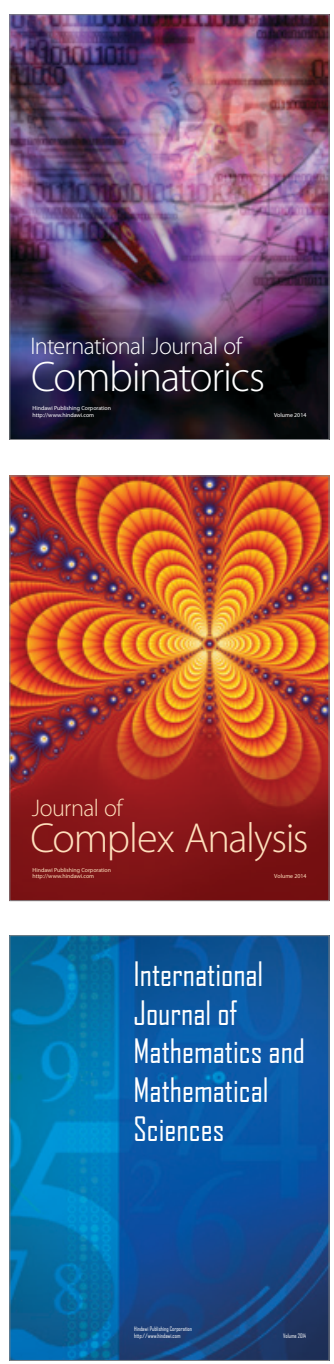
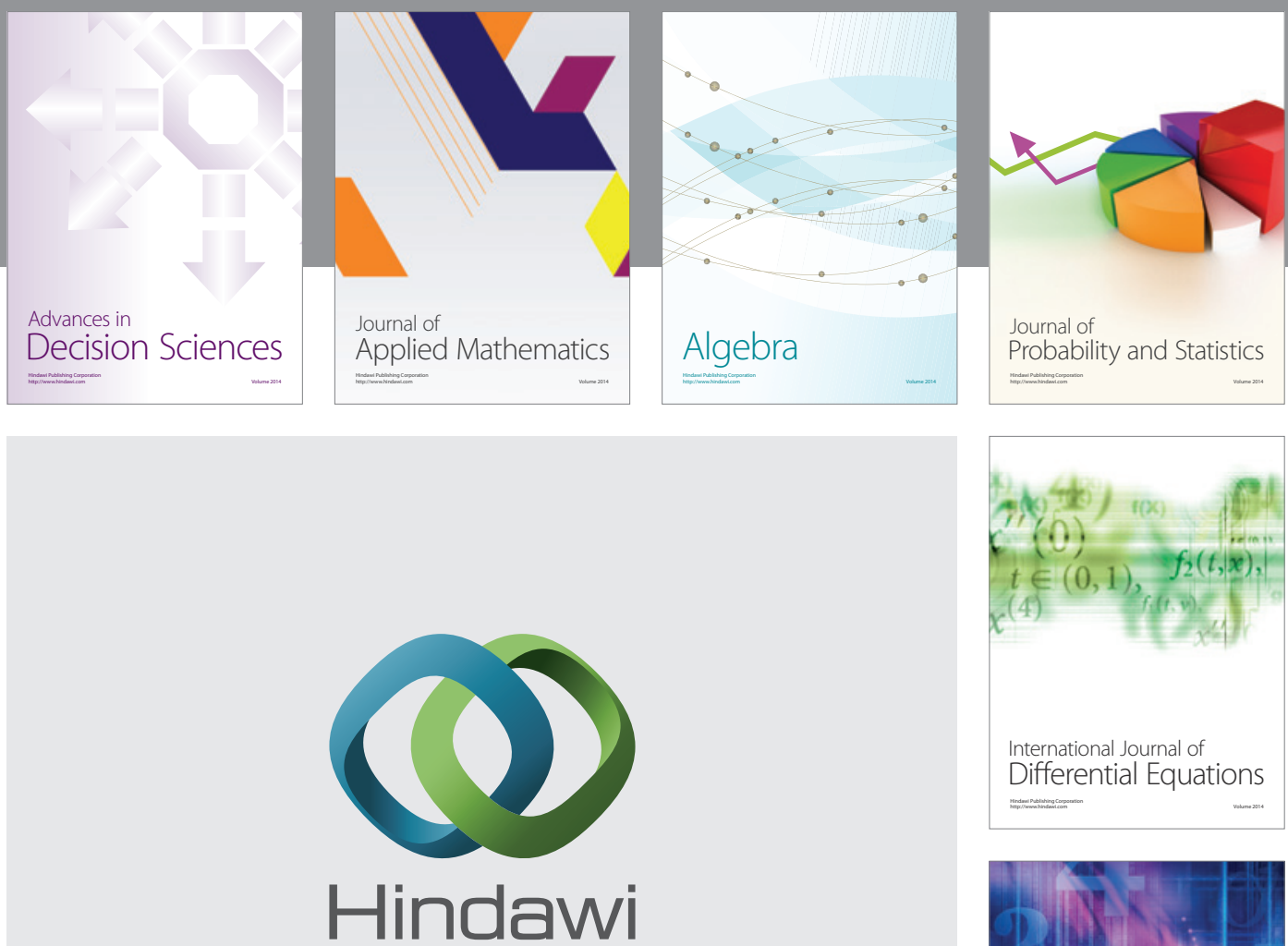

Submit your manuscripts at http://www.hindawi.com
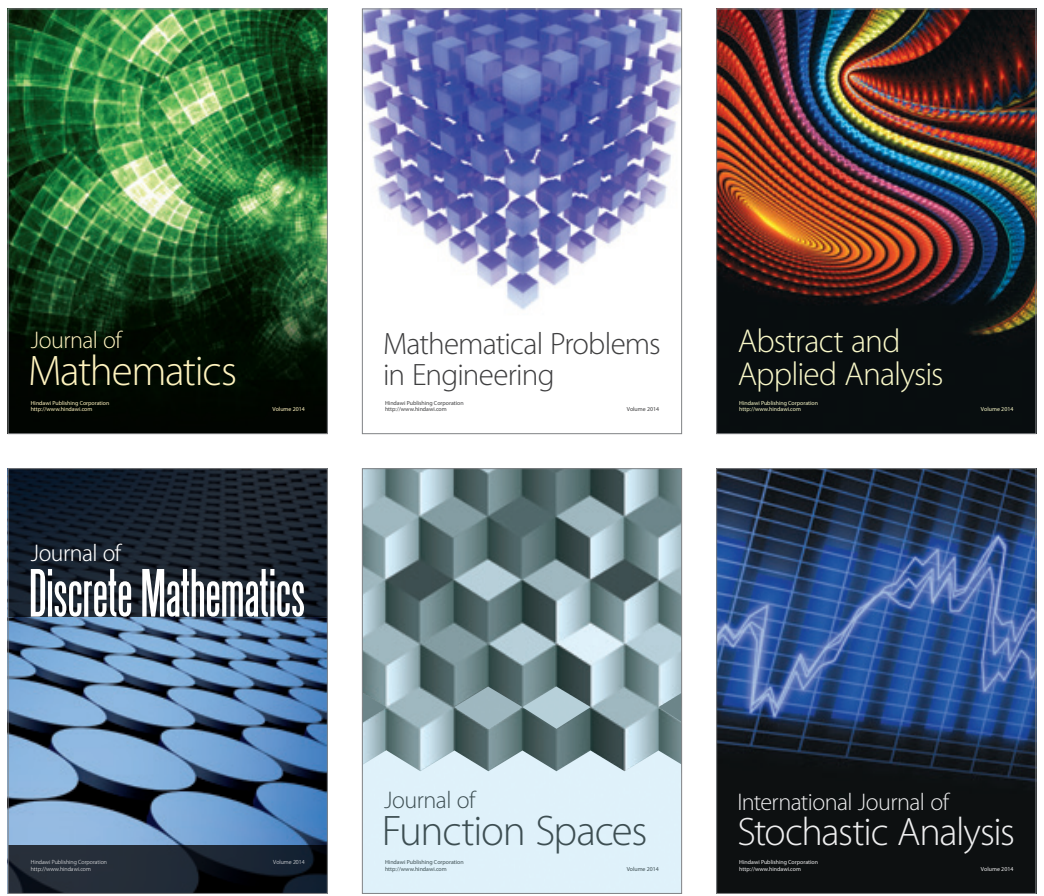

Journal of

Function Spaces

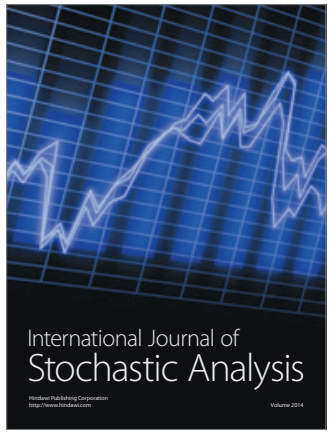

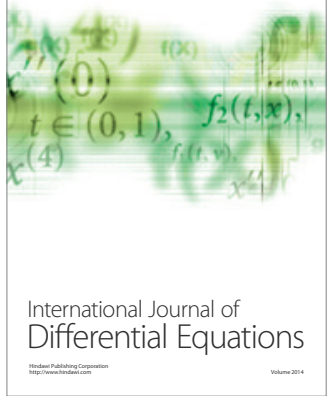
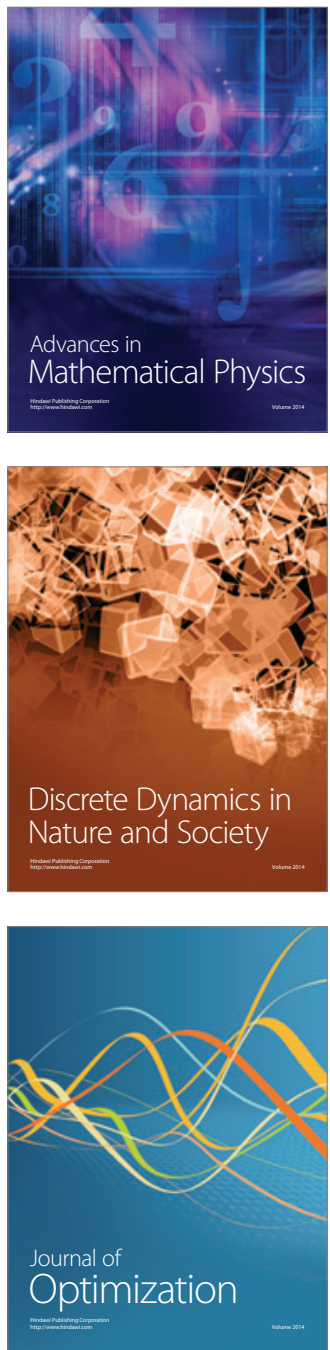